\section{Infectious Disease Pharmacotherapy Self Assessment}

Eiland LS, Ginsburg DB. American Society of Health-System Pharmacists, Bethesda, Maryland, 2015. Softcover, 208 pages. ISBN 978-1-58528-492-4. US\$40 (US\$35 for ASHP members).

Infectious Disease Pharmacotherapy Self Assessment is one of the latest volumes in the American Society of Health-System Pharmacists' Self Assessment series of books and e-books. In their preface, the authors state that the book is designed to provide patient-specific, case-based learning for clinicians at various stages of learning, from students to advanced practitioners.

The authors of this book are also editors of PharmPrep: ASHP's NAPLEX® Review (American Society of Health-System Pharmacists, 2011 [4th edition]), a case-based textbook designed to prepare pharmacy students for writing the North American Pharmacy Licensure Examination, and there is some overlap in cases between these 2 resources. The cases included in Infectious Disease Pharmacotherapy Self Assessment have been changed or updated from those in the infectious disease section of PharmPrep. All of these cases are based on real patients and were written by several contributing pharmacists.

The book is organized in 2 main parts. Part I consists of the patient cases, and part II presents answers to the 6 self-assessment questions that follow each case. There is also an appendix, which presents a glossary of abbreviations used throughout the cases. Part I comprises 33 cases divided into 9 sections for different types of infectious disease, such as bone and joint or skin and soft tissue infections and sexually transmitted diseases. The cases describe both adult and pediatric patients. Cases are designed at 3 levels of complexity: level 1 (beginner), level 2 (intermediate), and level 3 (advanced). The level 1 cases require only knowledge about the infectious disease involved in the particular case, whereas levels 2 and 3 require knowledge of broader infectious disease topics (such as antimicrobial resistance and stewardship). Part II provides detailed explanations of the answers to the self-assessment questions.
Unlike the Pharmacotherapy Self-Assessment Program of the American College of Clinical Pharmacy, this book does not provide any "prereading" for the topic of interest along with the case. Rather, readers are expected to attempt the self-assessment questions first, and then consult, as needed, the list of guidelines or relevant articles that is provided with the answers in part II. For those not familiar with a particular infectious disease topic, it would be helpful to have the list of recommended readings along with the case, so that they can familiarize themselves with the topic before attempting the selfassessment questions. Other criticisms of this book are similar to those for other US resources. The book requires familiarity with imperial units, as a conversion table to Système International (SI) units is not provided. Additionally, some of the answers to the questions do not reflect Canadian practices (e.g., the recommendation of daptomycin as an option for empiric treatment of gram-positive bacteremia, which reflects the higher prevalence of resistant gram-positive organisms, such as methicillin-resistant Staphylococcus aureus [MRSA], in the United States). As with any medical reference, some of the information in this book has become outdated. For example, some of the answers for the infective endocarditis cases are no longer correct, as guidelines have been updated since publication of this book.

Overall, Infectious Disease Pharmacotherapy Self Assessment is a well-organized book that can supplement learning about infectious diseases for both pharmacy trainees and more experienced clinicians. It can be used as a teaching aid to allow trainees to apply infectious disease concepts or as a workbook for clinicians to test their infectious disease knowledge.

Cesilia Nishi, BScPharm, ACPR, PharmD

Pharmacy

Vancouver General Hospital

Vancouver, British Columbia 